Pacific Journal of Mathematics

ON THE HYPERGROUP STRUCTURE OF CENTRAL $\Lambda(p$ 


\title{
ON THE HYPERGROUP STRUCTURE OF CENTRAL $\Lambda(p)$ SETS
}

\author{
GEORGE BENKE
}

Let $G$ be a compact group and let $\Gamma$ be the set of equivalence classes of the continuous irreducible unitary representations of $G$. For $\gamma \in \Gamma$ denote by $\chi_{\gamma}$ the character of $\gamma$, then for $E \subset \Gamma$ any function of the form $\sum_{j=1}^{n} a_{n} \chi_{\gamma_{n}}\left(\gamma_{1}, \cdots, \gamma_{n} \in E\right.$ and $\left.a_{1}, \cdots, a_{n} \in C\right)$ will be called a central $E$-polynomial, and the set of all such functions will be denoted ${ }^{2} \mathscr{T}_{E}$. A set $E \subset \Gamma$ is a central Sidon set when the norms \|\|$_{\infty}$ and \|\|$_{A}\left(\|f\|_{A}=\right.$ $\sum\left|a_{n}\right|$, where $\left.f=\sum a_{n} \chi_{\tau_{n}}\right)$ are equivalent on $z \mathscr{T}_{E}$, and it is a central $\Lambda(p)$ set when the norms \|\|$_{1}$ and \|\|$_{p}$ are equivalent on ${ }^{z} \mathscr{T}_{E}$. When $G$ is abelian the algebraic structure of $\Lambda(p)$ and Sidon set has been studied extensively. In this paper the structure of central $\Lambda(p)$ sets is investigated in terms of the hypergroup structure of $\Gamma$. In particular it is shown that central $\Lambda(p)(p>2)$ sets cannot contain arbitrarily long "arithmetic progressions."

1. Preliminary remarks. Following Helgason [2] we shall say that a set $S$ is hypergroup if to any pair $(\alpha, \beta)$ of elements from $S$ there corresponds a measure $\mu_{\alpha, \beta}$ on $S$. For $\Gamma$, a hypergroup structure is induced by the decomposition of tensor products. Thus if $\alpha, \beta \in$ $\Gamma \alpha \otimes \beta=\bigoplus_{\gamma \in \Gamma}[\gamma: \alpha \otimes \beta] \gamma$, where $[\gamma: \alpha \otimes \beta]$ is a nonnegative integer which is called the multiplicity of $\gamma$ in $\alpha \otimes \beta$, and the measure assigned to the pair $(\alpha, \beta)$ is the discrete measure whose mass at $\gamma$ is $[\gamma: \alpha \otimes$ $\beta]$. From the elementary properties of characters we write $\chi_{r_{1}} \cdots \chi_{r_{n}}=$ $\chi_{\gamma_{1} \otimes \cdots \otimes \gamma_{n}}=\sum_{\gamma \in \Gamma}\left[\gamma: \gamma_{1} \otimes \cdots \otimes \gamma_{n}\right] \chi_{\gamma}$. We shall denote by 1 the class of the trivial one dimensional representation, and by $\tilde{\gamma}$ the class containing the conjugates of representations in $\gamma$. All the basic facts about representations needed in this paper may be found in [3].

2. A necessary and sufficient condition for central $\Lambda(2 s)$. Although the condition we are about to give is cumbersome, it will allow us to get both necessary conditions and sufficient conditions which are reminiscent of conditions given by Rudin [6, Thm. 4.5] for the case where $G$ is the circle group.

THEOREM. Let $E \subset \Gamma$ and let $s$ be a natural number, then the following are equivalent.

(a) $E$ is a central $\Lambda(2 s)$ set.

(b) There exists a constant $B$ depending only on $E$ and $s$ such that for every choice of positive real numbers $a_{1}, \cdots, a_{N}$ and elements 
$\gamma_{1}, \cdots, \gamma_{N} \in E$ the inequality

$$
\sum_{r \in \Gamma}\left(\sum a_{k_{1}} \cdots a_{k_{s}}\left[\gamma: \gamma_{k_{1}} \otimes \cdots \otimes \gamma_{k_{s}}\right]\right)^{2} \leqq\left(B\left(\sum_{k=1}^{N} a_{k}^{2}\right)^{1 / 2}\right)^{2 s}
$$

holds, where the inner sum on the left is over all

$$
\left(k_{1}, \cdots, k_{s}\right) \in\{1, \cdots, N\}^{s} .
$$

Proof. The logarithmic convexity of the \|\|$_{p}$ norms shows that for $p>2$ a set $E$ is central $\Lambda(p)$ if \|\|$_{2}$ and \|\|$_{p}$ are equivalent on ${ }^{z} \mathscr{T}_{E}\left[6\right.$, Thm. 1.4]. Accordingly, we will work with the \|\|$_{2}$ and \|\|$_{2 s}$ norms.

Suppose that $E$ is a central $\Lambda(2 s)$ set, and choose positive real numbers $a_{1}, \cdots, a_{N}$ and $\gamma_{1}, \cdots, \gamma_{N} \in E$. Let $\mathscr{N}$ denote the set $\{1, \cdots$, $N\}^{s}$, denote elements in $\mathscr{N}$ by $\boldsymbol{k}$, write $a_{k_{1}}, \cdots, a_{k_{s}}$ as $a(k),\left(\gamma_{k_{1}}, \cdots, \gamma_{k_{s}}\right) \in$ $E^{s}$ as $\gamma(k)$ and $\gamma_{k_{1}} \otimes \cdots \otimes \gamma_{k_{s}}$ as $\gamma^{(k)}$ were $\left(k_{1}, \cdots, k_{s}\right)=k$. Define $f=\sum_{k=1}^{N} a_{k} \chi_{r_{k}}$, then

$$
\begin{aligned}
f^{s} & =\sum_{k \in \sim r} a(k) \chi_{\gamma(k)} \\
& =\sum_{k \in \sim r} a(k) \sum_{\gamma \in \Gamma^{\prime}}\left[\gamma: \gamma^{(k)}\right] \chi_{\gamma} \\
& =\sum_{\gamma \in \Gamma}\left(\sum_{k \in \sim r} a(k)\left[\gamma: \gamma^{(k)}\right]\right) \chi_{\gamma}
\end{aligned}
$$

Using the fact that the irreducible characters are an orthonormal family and $E$ is a central $\Lambda(2 s)$ set we have

$$
\|f\|_{2 s}^{2 s}=\left\|f^{s}\right\|_{2}^{2}=\sum_{\gamma \in \Gamma}\left(\sum_{k \in \mathcal{L}} a(k)\left[\gamma: \gamma^{(k)}\right]\right)^{2} \leqq\left(B\|f\|_{2}\right)^{2 s} .
$$

To show (b) implies (a), let $g=\sum_{k=1}^{N} b_{k} \chi_{\gamma_{k}}$ be any central $E$-polynomial. As before

$$
\begin{aligned}
\|g\|_{2 s}^{2 s} & =\sum_{\gamma \in \Gamma}\left|\sum_{k \in \sim r} b(k)\left[\gamma: \gamma^{(k)}\right]\right|^{2} \\
& \leqq \sum_{\gamma \in \Gamma}\left(\sum_{k \in \sim r}|b|(k)\left[\gamma: \gamma^{(k)}\right]\right)^{2}
\end{aligned}
$$

which by hypothesis is $\leqq\left(B\left(\sum_{k=1}^{N}\left|b_{k}\right|^{2}\right)^{1 / 2}\right)^{2 s}=\left(B\|g\|_{2}\right)^{2 s}$.

Corollary. Let $E \subset \Gamma$ be a central $\Lambda(2 s)$ set with constant $B$ so that $\|f\|_{2 s} \leqq B\|f\|_{2}$ for all central E-polynomials $f$, then for any finite subset $F \subset E$,

$$
\sum_{\gamma \in \Gamma}\left(\sum_{(\gamma) \in F^{s}}\left[\gamma: \gamma_{1} \otimes \cdots \otimes \gamma_{s}\right]\right)^{2} \leqq B^{2 s}(\operatorname{card} E)^{s} .
$$

Proof. In the theorem set $a_{1}=\cdots=a_{N}=1$. 
REMARK. A case where this criterion is violated in a very simple way is that of $G=S U(2)$. Here $\Gamma$ can be written as $\{\underline{1}, \underline{2}, \cdots\}$ and the Clebsch-Gordan [3, p. 135] formula shows that $\underline{n} \otimes \underline{n}=\underline{1} \oplus \underline{3} \oplus$ $\cdots \oplus \underline{2 n-1}$. So if $E$ is any set in $\Gamma$, take $F=\{\bar{n}\} \subset \bar{E}$, then

$$
\sum_{k=1}^{\infty}[\underline{k}: \underline{n} \otimes \underline{n}]^{2}=n
$$

and hence $\Gamma$ cannot contain any infinite central $4(4)$ sets. This fact has already been observed by Helgason [2, p. 789].

3. A sufficient condition for central $\Lambda(2 s)$. Let $F$ be any subset of $\Gamma$ and write as $(\gamma)$ the $s$-tuples $\left(\gamma_{1}, \cdots, \gamma_{s}\right) \in F^{s}$. Write $\otimes(\gamma)$ for $\gamma_{1} \otimes \cdots \otimes \gamma_{s}$, and for $(\gamma) \in F^{s}$ let $M((\gamma))$ stand for the set of irreducible components of $\otimes(\gamma)$. Furthermore, define

$$
r_{s}(F, \gamma)=\sum_{(\gamma) \in F^{s}}[\gamma: \otimes(\gamma)]^{2}
$$

Note that when $G$ is abelian $r_{s}(F, \gamma)$ is the number of ways we can write $\gamma=\gamma_{k_{1}} \otimes \cdots \otimes \gamma_{k_{s}}$ where $\gamma_{k_{j}} \in F$ and where a permutation of the same set of $\gamma_{k_{j}}$ 's is counted as a distinct partition of $\gamma$. The following corollary generalizes Rudin's result [6, Thm. 4.5(b)].

Corollary. Let $E \subset \Gamma$ and let $s$ be a natural number. If $E$ is the union of sets $E_{i}(i=1, \cdots, j)$ for which there exist constants $C_{i}$ and $D_{i}$ depending only on $E_{i}$ and $s$ such that

(i) $r_{s}\left(E_{i}, \gamma\right) \leqq C_{i}$ for all $\gamma \in \Gamma$ and

(ii) $\operatorname{card} M((\gamma)) \leqq D_{i}$ for all $(\gamma) \in E_{i}^{s}$ then

(a) $E$ is a central $\Lambda(2 s)$ set and

(b) $\|f\|_{2 s} \leqq\left(\sum_{i=1}^{j}\left(C_{i} D_{i}\right)^{1 / s}\right)^{1 / 2}\|f\|_{2}$ for all central E-polynomials $f$.

Proof. We show first that the $E_{i}$ are central $\Lambda(2 s)$ sets by applying the theorem of $\S 2$. Choose positive numbers $a_{1}, \cdots, a_{N}$ and $\gamma_{1}, \cdots$, $\gamma_{N} \in E_{i}$. Then

$$
\begin{aligned}
\sum_{\gamma \in \Gamma} & \left(\sum_{k \in \mathscr{S}} a(k)\left[\gamma: \gamma^{(k)}\right]\right)^{2} \\
& \leqq \sum_{k \in \mathscr{S}}\left(\sum_{k \in \mathscr{S}}\left[\gamma: \gamma^{(k)}\right]^{2}\right)\left(\sum_{k \in \mathscr{N}} a^{2}(k) \phi(\gamma, k)\right)
\end{aligned}
$$

where $\phi(\gamma, k)=1$ if $\gamma$ appears in the decomposition of $\gamma^{(k)}$ and $\phi=0$ otherwise. Observe that $\sum_{\gamma \in \Gamma} \phi(\gamma, \boldsymbol{k})=$ card $M(\gamma(\boldsymbol{k}))$ and so by hypothesis this sum is

$$
\leqq C_{i} D_{i} \sum_{k \in \mathscr{r}} a^{2}(k)=C_{i} D_{i}\left(\sum_{k=1}^{N} a_{k}^{2}\right)^{s} .
$$


Hence $E_{i}$ is a central $\Lambda(2 s)$ set and $\|f\|_{2 s} \leqq\left(C_{i} D_{i}\right)^{1 / 2 s}\|f\|_{2}$ for any central $f$.

Now suppose that the $E_{i}^{\prime}$ 's are disjoint, for if not they may be replaced by $E_{i}-\bigcup_{i=1}^{i-1} E_{l}$. If $f=\sum a_{r} \chi_{r}$ is a central $E$-polynomial then $f=f_{1}+\cdots+f_{j}$ where $f_{i}=\sum_{r \in E_{i}} a_{r} \chi_{r}$ and

$$
\begin{aligned}
\|f\|_{2 s} & \leqq \sum_{i=1}^{j}\left\|f_{i}\right\|_{2 s} \leqq \sum_{i=1}^{j}\left(C_{i} D_{i}\right)^{1 / 2 s}\left\|f_{i}\right\|_{2} \\
& \leqq\left(\sum_{i=1}^{j}\left(C_{i} D_{i}\right)^{1 / s}\right)^{1 / 2}\left(\sum_{i=1}^{j}\left\|f_{i}\right\|_{2}^{2}\right)^{1 / 2} \\
& =\left(\sum_{i=1}^{j}\left(C_{i} D_{i}\right)^{1 / s}\right)^{1 / 2}\|f\|_{2}
\end{aligned}
$$

since the $f_{i}$ are orthogonal.

REMARKS. (1) The condition (ii) of the previous corollary is also necessary. Take $F=\left\{\gamma_{1}, \cdots, \gamma_{s}\right\} \in E$ and apply the corollary in $\S 2$. Then we have

$$
\begin{aligned}
B^{2 s} s^{s} & \geqq \sum_{\gamma \in \Gamma}\left(\sum_{(\gamma) \in F^{s}}[\gamma: \otimes(\gamma)]\right)^{2} \\
& \geqq s ! \sum_{\gamma \in \Gamma}[\gamma: \otimes(\gamma)]=s ! \operatorname{card} M((\gamma))
\end{aligned}
$$

where $(\gamma)$ in the last two expressions is the $s$-tuple whose components are the elements of $F$.

(2) The condition (ii) is always satisfied when $\sup \{\operatorname{deg} \gamma \mid \gamma \in E\}=$ $P<\infty$. For if $(\gamma) \in E^{s}$, then the degree of $\otimes(\gamma)$ is not larger than $P^{s}$ and hence there can be at most $P^{s}$ elements in $M((\gamma))$.

4. The relationship between central Sidon and central $\Lambda(p)$ sets. A set $E \subset \Gamma$ will be called a central $\Lambda$ set if there exists a constant $C$ depending only on $E$ such that $\|f\|_{p} \leqq C p^{1 / 2}\|f\|_{2}$ for all $2<p<\infty$ and all central $E$-polynomials $f$. In the case of abelian groups, Rudin [7, p. 128] shows that every Sidon set is a central $\Lambda$ set. Using essentially the same technique Parker [5, p. 43] extends this result to central Sidon sets which have a uniform bound on the degrees of the representations in the set. Moreover, Parker [5, p. 73] shows by an example that some sort of condition is required; he gives an example of a central Sidon set which is not even central $\Lambda(4)$. Using essentially the same technique as Rudin and Parker we will characterize those central Sidon sets which are also central $\Lambda(2 s)$ or central 1 . An interesting consequence of this result is that a central Sidon set which is also central $\Lambda(p)$ for all $p$ must be a central $\Lambda$ set. It should be noted that sets which are central $\Lambda(p)$ for all $p<\infty$ need not in general be central $\Lambda$ sets, in fact such sets exist in every 
infinite abelian group [1, p. 788].

Theorem. Let $E \subset \Gamma$ be a central Sidon set.

(i) $E$ is central $\Lambda(2 s)$ if and only if there exists a constant $B$ depending on $E$ and $s$, so that $\left\|\chi_{r}\right\|_{2 s} \leqq B$ for all $\gamma \in E$.

(ii) $E$ is central $A$ if and only if there exists a constant $B$ depending only on $E$ such that $\left\|\chi_{r}\right\|_{2 s} \leqq B$ for all $\gamma \in E$ and $s=1,2, \cdots$.

Proof. Since $\left\|\chi_{r}\right\|_{2}=1$ for all $\gamma \in \Gamma$ we clearly have the "only if" parts of (i) and (ii).

Suppose $E$ is a central Sidon set and we have a constant $B$ as in (i). Let $f=\sum_{n=1}^{N} a_{n} \chi_{r_{n}}$ be a central $E$-polynomial. Let

$$
\Omega=\Pi_{1}^{N}\{-1,1\}
$$

with the operation of coordinatewise multiplication and let $\varepsilon_{n}: \Omega \rightarrow$ $\{-1,1\}$ be projection onto the $n$th coordinate. Since $E$ is a central Sidon set, for every $\omega \in \Omega$ there exists a central measure $\mu_{\omega}$ on $G$ such that $\hat{\mu}_{\omega}\left(\gamma_{n}\right)=\varepsilon_{n}(\omega) I_{d_{\gamma_{n}}}(n=1, \cdots, N)$ and $\left\|\mu_{\omega}\right\|_{1} \leqq C$ where $C$ depends only on $E$ [5, p. 27]. We have

$$
\begin{aligned}
\|f\|_{2 s}^{2 s} & =\left\|\mu_{\omega} * \mu_{\omega} * f\right\|_{2 s}^{2 s} \leqq\left\|\mu_{\omega}\right\|_{1}^{2 s}\left\|\mu_{\omega} * f\right\|_{2 s}^{2 s} \\
& \leqq C^{2 s} \int_{G}\left|\sum_{n=1}^{N} a_{n} \chi_{\gamma_{n}}(x) \varepsilon_{n}(\omega)\right|^{2 s} d x
\end{aligned}
$$

Integrating both sides of the inequality over $\Omega$ and using Fubini's theorem and the inequality

$$
\left(\int_{\Omega}\left|\sum_{n=1}^{N} b_{n} \varepsilon_{n}(\omega)\right|^{2 s} d \omega\right)^{1 / 2 s} \leqq 2 \sqrt{s}\left(\sum_{n=1}^{N}\left|b_{n}\right|^{2}\right)^{1 / 2}
$$

whose proof is the same as that of $[8,8.4$, p. 213], we have

$$
\begin{aligned}
\|f\|_{2 s}^{2 s} & \leqq C^{2 s} 2^{2 s} s^{s} \int_{G}\left(\sum_{n=1}^{N}\left|a_{n}\right|^{2}\left|\chi_{\gamma_{n}}(x)\right|^{2}\right)^{s} d x \\
& =(2 \sqrt{s} C)^{2 s} \sum\left|a_{n_{1}}{ }^{2}\right| \cdots\left|a_{n_{s}}\right|^{2} \int_{G}\left|\chi_{\gamma_{n_{1}}}\right|^{2} \cdots\left|\chi_{\gamma_{n_{s}}}\right|^{2} d x
\end{aligned}
$$

where the sum is over all $\left(n_{1}, \cdots, n_{s}\right) \in\{1, \cdots, N\}^{s}$. By Hölder's inequality this expression is

$$
\begin{aligned}
& \leqq(2 \sqrt{s} C)^{2 s} \sum\left|a_{n_{1}}\right|^{2} \cdots \mid a_{n_{s}}{ }^{2} I_{j=1}^{s}\left(\int_{G} \mid \chi_{r_{n_{j}}}{ }^{2 s} d x\right)^{2 / 2 s} \\
& \leqq(2 \sqrt{s} C)^{2 s}\left(\sum_{n=1}^{N}\left|a_{n}\right|^{2}\right)^{s} B^{2 s}
\end{aligned}
$$

that is, $\|f\|_{2 s} \leqq(C B \sqrt{2}) \sqrt{2 s}\|f\|_{2}$.

REMARKs. (1) Since deg $\gamma=\left\|\chi_{r}\right\|_{\infty}=\lim _{s \rightarrow \infty}\left\|\chi_{r}\right\|_{2 s}$, (ii) is a restate- 
ment of Parker's result.

(2) The following are equivalent.

(a) There exists a constant $B$ depending only on $E$ and s so that $\left\|\chi_{\gamma}\right\|_{2 s} \leqq B$ for all $\gamma \in E$.

(b) There exist constants $C$ and $D$ depending only on $E$ and $s$ so that

(i) $[\sigma: \otimes(\gamma)] \leqq C$ for all $\sigma \in \Gamma$ and $\gamma \in E$ where $(\gamma)$ is the $s$-tuple whose components are $\gamma$, and

(ii) card $M((\gamma)) \leqq D$ for all $\gamma \in E$.

The orthogonality of the characters gives

$$
\begin{aligned}
\left\|\chi_{\gamma}\right\|_{2 s}^{2 s} & =\int_{G} \chi_{\gamma}^{s} \bar{\chi}_{\gamma}^{s} d x \\
& =\int_{G}\left(\sum_{v \in \Gamma}[\sigma: \otimes(\gamma)] \chi_{\sigma}\right)\left(\sum_{\nu \in \Gamma}[\nu: \otimes(\gamma)] \bar{\chi}_{\nu}\right) d x \\
& =\sum_{(\sigma, \nu) \in \Gamma^{\prime} \times \Gamma}[\sigma: \otimes(\gamma)][\nu: \otimes(\gamma)] \int_{G} \chi_{\gamma} \bar{\chi}_{\nu} d x \\
& =\sum_{\sigma \in M((\gamma))}[\sigma: \otimes(\gamma)]^{2} .
\end{aligned}
$$

Since the terms in this last sum are positive we have the equivalence of (a) and (b).

5. Product groups and lacunarv projections. Let $G_{\alpha}, \alpha \in I$ be a collection of compact groups with dual objects $\Gamma_{\alpha}$. Let $G=\prod_{\alpha \in I} G_{\alpha}$ be the complete direct product and $\Gamma=\prod_{\alpha \in I}^{*} \Gamma_{\alpha}$ be the incomplete direct product. Then $\Gamma$ is the dual object of $G$ and the operations are all the obvious coordinatewise ones [3, p. 27]. Let $\sigma_{\alpha} \in \Gamma_{\alpha}$ and let $\pi_{\alpha}$ : $G \rightarrow G_{\alpha}$ be the projection onto the $\alpha^{\prime}$ th coordinate, then $\sigma_{\alpha} \circ \pi_{\alpha} \in \Gamma$. Write $\sigma_{\alpha}^{j}$ for the $j$-fold tensor product of $\sigma_{\alpha}$ in $\Gamma_{\alpha}$ and let $M\left(\sigma_{\alpha}^{j}\right)$ be the set of irreducible components of $\sigma_{\alpha}^{j}$ in $\Gamma_{\alpha}$.

Theorem. Let $G$ and $\Gamma$ be as above and consider $E=\left\{\gamma_{\alpha}=\right.$ $\left.\pi_{\alpha} \circ \sigma_{\alpha} \mid \alpha \in I\right\}$. A necessary and sufficient condition that $E$ be a central $\Lambda(2 s)$ set is that there exist constants $K$ and $L$ both depending only on $s$ and the set $\left\{\sigma_{\alpha} \mid \alpha \in I\right\}$ so that

(a) $\left[\tau_{\alpha}: \sigma_{\alpha}^{s}\right] \leqq L$ for all $\tau_{\alpha} \in \Gamma_{\alpha}$ and $\alpha \in I$, and

(b) $\operatorname{card} M\left(\sigma_{\alpha}^{s}\right) \leqq K$ for all $\alpha \in I$.

Proof. Parker [5, p. 70] shows that $E$ is a central Sidon set, hence by the theorem in $\S 4$ we need a uniform bound on $\left\|\chi_{\gamma_{\alpha}}\right\|_{2 s}$ as $\alpha$ ranges over $I$. Since Haar measure on $G$ is just the product of the Haar measures on the $G_{\alpha}$, we have $\left\|\chi_{\gamma_{\alpha}}\right\|_{2 s}=\left\|\chi_{\sigma_{\alpha}}\right\|_{2 s}$ but by remark (2) of $\S 4$ this is equivalent to the conditions (a) and (b). 
RemarK. If $\sup \left\{\operatorname{deg} \sigma_{\alpha} \mid \alpha \in I\right\}=P<\infty$, then $E$ is a central $\Lambda(2 s)$ set.

6. Intersections with arithmetic progressions. Let $\sigma \in \Gamma$ and let $N$ be a natural number, we define the arithmetic progression of length $N$ generated by $\sigma$ to be

$$
A(\sigma, N)=\bigcup_{j=1}^{N} M\left(\sigma^{j}\right)
$$

where $\sigma^{j}$ is the $j$-fold tensor product of $\sigma$.

THEOREM. Let $E$ be a central $\Lambda(p)$ set $(p>2)$ with constant $B$ so that $\|f\|_{p} \leqq B\|f\|_{2}$ for all central E-polynomials $f$. Let $\sigma \in \Gamma$, then

$$
\operatorname{card}(A(\sigma, N) \cap E)=0\left(N^{4(\operatorname{deg} \sigma)^{2} / p}\right) \text { as } N \longrightarrow \infty \text {. }
$$

Proof. Choose $\varepsilon$ and let $D_{2 N}^{o}=\sum_{\gamma \in A(\sigma, 2 N)} d_{\gamma} \chi_{\gamma}$ and

$$
F_{2 N}^{s}=\left|D_{2 N}^{\sigma}\right|^{2} /\left(\sum_{\gamma \in A(\sigma, 2 N)} d_{\gamma}^{2}\right)
$$

so

$$
\begin{aligned}
F_{2 N}^{o} & =\left(\sum d_{\gamma} \chi_{\gamma}\right)\left(\sum d_{\nu} \bar{\chi}_{\nu}\right) /\left(\sum d_{\gamma}^{2}\right) \\
& =\left(\sum_{\zeta \in \Gamma}\left(\sum d_{\gamma} d_{\nu}[\zeta: \gamma \otimes \bar{\nu}]\right) \chi_{\zeta}\right) /\left(\sum d_{\gamma}^{2}\right)
\end{aligned}
$$

where the inner sum is over all $(\gamma, \nu) \in A(\sigma, 2 N) \times A(\sigma, 2 N)$. If we write $F_{2 N}^{o}=\sum_{\zeta \in \Gamma} d_{\zeta} \alpha\left(F_{2 N}^{o}, \zeta\right) \chi_{\zeta}$ then Mayer [4, p. 688] shows that for all $N$ sufficiently large and $\zeta \in A(\sigma, N)$

$$
\alpha\left(F_{2 N}^{o}, \zeta\right) \geqq r_{\sigma}(N) / d_{\zeta} r_{\sigma}(2 N)
$$

where $r_{\sigma}$ is a polynomial of degree $\leqq d_{\sigma}^{2}$. Choose $\eta>0$ small enough so that $\left(2^{-(\operatorname{deg} \sigma) 2}-\eta\right)^{-1} \leqq 2^{(\operatorname{deg} \sigma)^{2}}+\varepsilon$. Then for this $\eta$ and $\zeta \in A(\sigma, N)$ we have for $N$ sufficiently large that

$$
\alpha\left(F_{2 N}^{o}, \zeta\right) \geqq\left(2^{-(\operatorname{deg} \sigma)^{2}}-\eta\right) / d_{\zeta} .
$$

We also have $\left\|F_{2 N}^{s}\right\|_{2} \leqq\left\|F_{2 N}^{o}\right\|_{\infty}=\left(D_{2 N}^{\sigma}(e)\right)^{2} /\left(\sum d_{\gamma}^{2}\right)$, and since $\chi_{r}(e)=d_{\gamma}$ we have

$$
\left\|F_{2 N}^{j}\right\|_{2} \leqq \sum_{\gamma \in A(\sigma, 2 N)} d_{\gamma}^{2}=r_{\sigma}(N)
$$

for all $N$ sufficiently large as shown in [4, p. 687]. Hence

$$
\left\|F_{2 N}^{a}\right\|_{2} \leqq K N^{(\operatorname{deg} \sigma)^{2}}
$$

for all $N$ sufficiently large. Let $f=\sum_{\zeta \in E \cap A(a, N)} \chi_{\zeta}$, define $\alpha(f, \zeta)$ so that $f=\sum_{\zeta \in \Gamma} d_{\zeta} \alpha(f, \zeta) \chi_{\zeta}$, and suppose $N$ is large enough to satisfy (1) 
and (2).

Then

$$
\begin{aligned}
\operatorname{card} & (E \cap A(\sigma, N))=\sum_{\zeta \in \Gamma} d_{\zeta} \alpha(f, \zeta) \\
& =\frac{1}{\left(2^{-(\operatorname{deg} \sigma)^{2}}-\eta\right)} \sum_{\zeta \in \Gamma} d_{\zeta} \alpha(f, \xi)\left(2^{-(\operatorname{deg} \sigma)^{2}}-\eta\right) \\
& \leqq \frac{1}{\left(2^{-(\operatorname{deg} \sigma)^{2}}-\eta\right)} \sum_{\zeta \in \Gamma} d_{\zeta} \alpha(f, \zeta) d_{\zeta} \alpha\left(F_{2 N}^{\sigma}, \zeta\right) \\
& =\left(2^{-(\operatorname{deg} \sigma)^{2}}-\eta\right)^{-1} \int_{G} f(x) F_{2 N}^{\sigma}(x) d x \\
& \leqq\left(2^{-(\operatorname{deg} \sigma)^{2}}-\eta\right)^{-1}\|f\|_{p}\left\|F_{2 N}^{\sigma}\right\|_{q} .
\end{aligned}
$$

The logarithmic convexity of the \|\|$_{p}$ norms gives \|\|$_{q} \leqq\|\|_{1}^{(2-q) / q}\|\|_{2}^{2 / p}$. Using this fact and the hypothesis that $E$ was a central $\Lambda(p)$ set, the last expression is

$$
\leqq B\left(2^{-(\operatorname{deg} \sigma)^{2}}+\varepsilon\right)\|f\|_{2}\left\|F_{2 N}^{o}\right\|_{1}^{(2-q) / q}\left\|F_{2 N}^{o}\right\|_{2}^{2 / p} .
$$

Note that $\|f\|_{2}=(\operatorname{card}(A(\sigma, N) \cap E))^{1 / 2}$ and $\left\|F_{2 N}^{\sigma}\right\|_{1}=\hat{F}_{2 N}^{o}(1)=1$, so that by (2) we have

$$
(\operatorname{card}(A(\sigma, N) \cap E))^{1 / 2} \leqq B\left(2^{(\mathrm{deg} \sigma)^{2}}+\varepsilon\right)\left(K N^{(\mathrm{deg} \sigma)^{2}}\right)^{2 / p}
$$

for all $N$ sufficiently large, the size of $N$ depending only on $\sigma$ and $\varepsilon$.

Corollary. Let $E$ be a central $\Lambda$ set, and let $\sigma \in \Gamma$. Then

$$
\operatorname{card}(A(\sigma, N) \cap E)=0(\log N) \text {. }
$$

Proof. For a central $A$ set we may take $B=C p^{1 / 2}$ where $C$ depends only on $E$. In the last inequality of the previous proof, set $p=4 \log \left(K N^{(\operatorname{deg} \sigma)^{2}}\right)$, then

$$
\operatorname{card}(A(\sigma, N) \cap E) \leqq\left(2^{(\operatorname{deg} \sigma)^{2}}+\varepsilon\right)^{2} C^{2} e 4 \log \left(K N^{(\operatorname{deg} \sigma)^{2}}\right)
$$

for all $N$ sufficiently large.

\section{REFERENCES}

1. R. E. Edwards, E. Hewitt, and K. A. Ross, Lacunarity for compact groups, Indiana J. Math., 21 (1972), 787-806.

2. S. Helgason, Lacunary Fourier series on noncommutative groups, Proc. Amer. Math. Soc., 9 (1958), 782-780.

3. E. Hewitt and K. A. Ross, Abstract Harmonic Analysis, II, Springer-Verlag, 1970.

4. R. Mayer, Summation of Fourier series on compact groups, Amer. J. Math., 89 (1967), 661-692.

5. W. A. Parker, Central Sidon sets and central $\Lambda(p)$ sets, Thesis, University of Oregon, 1970.

6. W. Rudin, Trigonometric series with gaps, J. Math. Mech., 9 (1960), 203-228. 
7. W. Rubin, Fourier Analysis on Groups, Interscience Publishers, New York, 1962.

8. A. Zygmund, Trigonometric Series, I, Cambridge University Press, London, 1968.

Received September 27, 1972 and in revised form January 31, 1973.

GEORGETOWN UNIVERSITY 



\section{PACIFIC JOURNAL OF MATHEMATICS}

\section{EDITORS}

RICHARD ARENS (Managing Editor) University of California

Los Angeles, California 90024

R. A. BeaUmont

University of Washington Seattle, Washington 98105
J. DUGUNDJI*

Department of Mathematics University of Southern California Los Angeles, California 90007

D. Gilbarg and J. Milgram Stanford University

Stanford, California 94305

\section{ASSOCIATE EDITORS}

E. F. BECKENBACH

B. H. NeUmanN

F. WOLF

K. YoSHIDA

\section{SUPPORTING INSTITUTIONS}

\section{UNIVERSITY OF BRITISH COLUMBIA CALIFORNIA INSTITUTE OF TECHNOLOGY UNIVERSITY OF CA.LIFORNIA MONTANA STATE UNIVERSITY UNIVERSITY OF NEVADA NEW MEXICO STATE UNIVERSITY OREGON STATE UNIVERSITY UNIVERSITY OF OREGON OSAKA UNIVERSITY}

UNIVERSITY OF SOUTHERN CALIFORNIA STANFORD UNIVERSITY UNIVERSITY OF TOKYO UNIVERSITY OF UTAH WASHINGTON STATE UNIVERSITY UNIVERSITY OF WASHINGTON AMERICAN MATHEMATICAL SOCIETY NAVAL WEAPONS CENTER

* C. R. DePrima California Institute of Technology, Pasadena, CA 91109, will replace J. Dugundji until August 1974. 


\section{Pacific Journal of Mathematics}

\section{Vol. 50, No. $1 \quad$ September, 1974}

Gail Atneosen, Sierpinski curves in finite 2-complexes.............. 1

Bruce Alan Barnes, Representations of $B^{*}$-algebras on Banach spaces .... 7

George Benke, On the hypergroup structure of central $\Lambda(p)$ sets ....... 19

Carlos R. Borges, Absolute extensor spaces: a correction and an

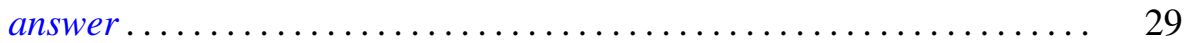

Tim G. Brook, Local limits and tripleability .................. 31

Philip Throop Church and James Timourian, Real analytic open maps .... 37

Timothy V. Fossum, The center of a simple algebra ............... 43

Richard Freiman, Homeomorphisms of long circles without periodic

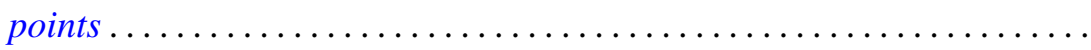

B. E. Fullbright, Intersectional properties of certain families of compact

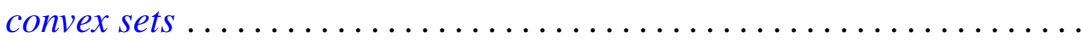

Harvey Charles Greenwald, Lipschitz spaces on the surface of the unit

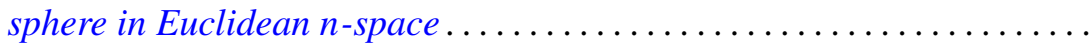

Herbert Paul Halpern, Open projections and Borel structures for

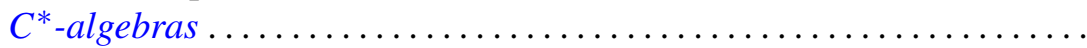

Frederic Timothy Howard, The numer of multinomial coefficients divisible

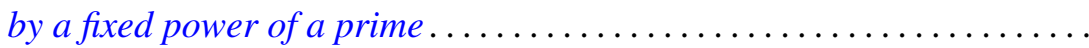

Lawrence Stanislaus Husch, Jr. and Ping-Fun Lam, Homeomorphisms of manifolds with zero-dimensional sets of nonwandering points........ 109

Joseph Edmund Kist, Two characterizations of commutative Baer rings ...

Lynn McLinden, An extension of Fenchel's duality theorem to saddle functions and dual minimax problems ................

Leo Sario and Cecilia Wang, Counterexamples in the biharmonic classification of Riemannian 2-manifolds...

Saharon Shelah, The Hanf number of omitting complete types ...

Richard Staum, The algebra of bounded continuous functions into a

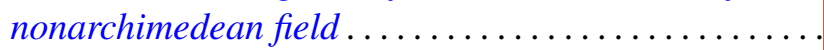

James DeWitt Stein, Some aspects of automatic continuity ..

Tommy Kay Teague, On the Engel margin

John Griggs Thompson, Nonsolvable finite groups all of whose local subgroups are solvable, $V \ldots \ldots \ldots \ldots \ldots \ldots \ldots \ldots$

Kung-Wei Yang, Isomorphisms of group extensions 